\title{
ON SOME FUNCTIONAL EQUATIONS FROM ADDITIVE AND NON-ADDITIVE MEASURES-I
}

\author{
by PL. KANNAPPAN
}

(Received 20th July 1978)

\section{Introduction}

It is known that while the Shannon and the Rényi entropies are additive, the measure entropy of degree $\beta$ proposed by Havrda and Charvat (7) is non-additive. Ever since Chaundy and McLeod (4) considered the following functional equation

$$
\begin{gathered}
\sum_{i=1}^{m} \sum_{j=1}^{n} f\left(x_{i} y_{j}\right)=\sum_{i=1}^{m} f\left(x_{i}\right)+\sum_{j=1}^{n} f\left(y_{j}\right) \\
\left(x_{i}, y_{j} \geqq 0, \quad \sum x_{i}=1=\Sigma y_{j}\right)
\end{gathered}
$$

which arose in statistical thermodynamics, (1.1) has been extensively studied $(\mathbf{1}, \mathbf{5}, \mathbf{6}, \mathbf{8})$. From the algebraic properties of symmetry, expansibility and branching of the entropy (viz. Shannon entropy $H_{n}$, etc.) one obtains the sum representation

$$
\left(\text { viz. } H_{n}(P)=\sum_{i=1}^{n} f\left(P_{i}\right), P=\left(p_{1}, \ldots, p_{n}\right) \text { with } \sum_{i=1}^{n} p_{i}=1\right)
$$

which with the property of additivity yields the functional equation $(1.1),(9,10)$.

If we write

$$
g(p)=\frac{p^{\beta}-p}{2^{1-\beta}-1}, \quad p \in[0,1], \quad\left(\beta \neq 1,0^{\beta}=0,1^{\beta}=1\right)
$$

then the entropy of degree $\beta$ (7)

$$
H_{n}^{\beta}(P)=\frac{\sum_{i=1}^{n} p_{i}^{\beta}-1}{2^{1-\beta}-1}
$$

takes the sum form

$$
H_{n}^{\beta}(P)=\sum_{i=1}^{n} g\left(p_{i}\right)
$$

and $g$ satisfies the functional equation

$$
\begin{aligned}
\sum_{i=1}^{m} \sum_{j=1}^{n} g\left(x_{i} y_{j}\right)= & \sum_{i=1}^{m} g\left(x_{i}\right)+\sum_{j=1}^{n} g\left(y_{j}\right)+c \sum_{i=1}^{m} g\left(x_{i}\right) \cdot \sum_{j=1}^{n} g\left(y_{j}\right) \\
& \left(x_{i}, y_{j} \geqq 0, \quad \Sigma x_{i}=1=\Sigma y_{j}\right)
\end{aligned}
$$

similar to (1.1) with $c=\left(2^{1-\beta}-1\right)$, treated in $(3,11)$. 
The equations (1.1) and (1.3) were studied under various hypotheses: such as the equations holding for all positive integers $m, n$ or (1.1) holding for only one pair $m=2$, $n=3$ and under some regularity condition on $f$ or $g$ etc. In this paper we propose to solve the functional equations (1.1) and (1.3) holding for some (arbitrary but) fixed pair $(m, n)$ when the functions involved are Lebesgue measurable using a different technique and to show that the solutions depend upon the pair $(m, n)$ and to obtain in the case of (1.3) more solutions than hitherto known.

\section{Auxiliary result}

Let $\Gamma_{n}=\left\{P=\left(p_{1}, \ldots, p_{n}\right): p_{i} \geqq 0, \Sigma p_{i}=1\right\}$. The measurable solutions of the functional equations (1.1) and (1.3) are obtained in Section 3 based on the solution of the following functional equation

$$
\sum_{i=1}^{m} \sum_{j=1}^{n} F\left(x_{i}, y_{j}\right)=0
$$

$\left(X=\left(x_{i}\right) \in \Gamma_{m}, Y=\left(y_{j}\right) \in \Gamma_{n}\right)$ holding for some fixed pair of integers $m, n \geqq 3$. Now, we prove the following lemma:

Lemma 1. Let $F:[0,1] \times[0,1] \rightarrow \boldsymbol{R}$ (reals), be measurable in each variable and satisfy the functional equation (2.1) for fixed $m, n(\geqq 3)$. Then $F$ is given by

$$
F(x, y)=F(0, y)(1-m x)+F(x, 0)(1-n y)-F(0,0)(1-m x)(1-n y) \text {. }
$$

Proof. For fixed $\left(y_{1}, y_{2}, \ldots, y_{n}\right)=\boldsymbol{y} \in \Gamma_{n}$, define

$$
h(x)=\sum_{j=1}^{n} F\left(x, y_{j}\right), \quad x \in[0,1] .
$$

Then $h$ is measurable and (2.1) can be rewritten as

$$
\sum_{i=1}^{m} h\left(x_{i}\right)=0 \quad\left(x_{i} \geqq 0, \Sigma x_{i}=1\right)
$$

From (12), it follows that (for $m \geqq 3$ )

$$
h(x)=a(1-m x) \quad(x \in[0,1])
$$

where $a$ is a function of $y_{1}, \ldots, y_{n}$.

Using (2.3) and (2.5), we get

so that

$$
\begin{aligned}
& \sum_{j=1}^{n} F\left(x, y_{j}\right)=a\left(y_{1}, \ldots, y_{n}\right)(1-m x), \\
& \sum_{j=1}^{n} F\left(0, y_{j}\right)=a\left(y_{1}, \ldots, y_{n}\right)
\end{aligned}
$$

$$
\sum_{j=1}^{n} l_{x}\left(y_{j}\right)=0
$$


where

$$
l_{x}(y)=F(x, y)-F(0, y)(1-m x), \text { for } y \in[0,1],
$$

is measurable. Again, from (12), it follows that (for $n \geqq 3$ ),

$$
l_{x}(y)=b(x)(1-n y), \text { for } y \in[0,1] .
$$

From (2.7) and (2.8) results, $b(x)=l_{x}(0)=F(x, 0)-F(0,0)(1-m x)$ so that we obtain

$$
F(x, y)=F(0, y)(1-m x)+F(x, 0)(1-n y)-F(0,0)(1-m x)(1-n y)
$$

which is precisely (2.2). This proves Lemma 1.

Remark 1. Suppose the equation (2.1) holds for 3 pairs $(m, n),(\gamma, s)$ and $(p, q)$. Then

$$
\left.\begin{array}{r}
F(0, y)(\gamma-m) x+F(x, 0)(s-n) y-F(0,0)[(\gamma-m) x+(s-n) y \\
F(0, y)(p-m) x+F(x, 0)(q-n) y-F(0,0)[(p-m) x+(q-n) y-m) x y=0 \\
-(p q-m n) x y]=0
\end{array}\right\}
$$

From (2.9), it is easy to see that, $F(0, y)$ and $F(x, 0)$ are given by

$$
\left.\begin{array}{l}
F(0, y)=F(0,0)\left[1-\frac{p q s-p q n-m n s-\gamma s q+\gamma s n+m n q}{(\gamma-m)(q-n)-(p-m)(s-n)} y\right] \\
F(x, 0)=F(0,0)\left[1-\frac{p q \gamma-p q m-m n \gamma-\gamma s p+\gamma s n+m n p}{(s-n)(p-m)-(\gamma-m)(q-n)} x\right],
\end{array}\right\}
$$

provided $(\gamma-m)(q-n)-(s-n)(p-m) \neq 0$.

(2.10) gives explicit values for $F(0, y)$ and $F(x, 0)$ in terms of $x, y, m, n, \gamma, s, p, q$.

It also follows from (2.9) that,

$$
F(x, y)=F(0, y)(1-m x)=f(y)(1-m x)
$$

where $f$ is arbitrary, when $(2.1)$ holds for the 2 pairs $(m, n)$ and $(m, s)$;

$$
F(x, y)=F(x, 0)(1-n y)=g(x)(1-n y)
$$

where $g$ is arbitrary, when (2.1) holds for the 2 pairs $(m, n)$ and $(\gamma, n)$;

$$
F(x, y)=(1-m x)(1-n y) \text {, }
$$

when (2.1) holds for the 3 pairs $(m, n),(\gamma, n)$ and $(m, s)$.

From (2.11), it follows that, when (2.1) holds for all pairs $(m, n)$, then

$$
F(x, y)=0
$$

(a result found in (2), under the condition of continuity of $F$ ).

\section{Solutions of the equations (1.1) and (1.3)}

Let $f, g:[0,1] \rightarrow R$, be measurable and satisfy the functional equations (1.1) and (1.3), for a fixed pair $m, n(\geqq 3)$ where $c$ is a constant. When $c=0,(1.3)$ and (1.1) are one and the same. We follow the convention $0 \log 0=0,0^{\beta}=0,1^{\beta}=1$. 
By letting

$$
F(x, y)=g(x y)-y g(x)-x g(y)-c g(x) g(y),
$$

for $x, y \in[0,1]$, we see that $F$ is measurable in each variable and that (1.3) takes the form (2.1). Thus by Lemma 1 and (3.1), we obtain

$$
\begin{aligned}
g(x y)= & y g(x)+x g(y)+c g(x) g(y) \\
& +g(0)(1-y-c g(y))(1-m x)+g(0)(1-x-c g(x))(1-n y) \\
& -g(0)(1-c g(0))(1-m x)(1-n y)
\end{aligned}
$$

for $x, y \in[0,1]$, where $c$ is a constant.

First, let us consider the case when $c=0$ (i.e the equation (1.1)). Then (3.2) becomes

where

$$
g(x y)=y g(x)+x g(y)+d[1-x-y+(m+n-m n) x y]
$$

i.e.

$$
d=g(0)
$$

$$
g(x y)-d-d(m+n-m n) x y=x(g(y)-d)+y(g(x)-d)
$$

i.e.

where

$$
h(x y)=x k(y)+y k(x), \quad(x, y \in[0,1])
$$

and

$$
h(x)=g(x)-d(1+m+n-m n) x,
$$

$$
k(x)=g(x)-d
$$

are measurable functions.

Now, (3.4) can be rewritten as

$$
\left.\left.\frac{h(x y)}{x y}=\frac{k(y)}{y}+\frac{k(x)}{x}, \quad x, y \in\right] 0,1\right],
$$

a Pexider equation, so that, the measurability of the functions imply,

$$
k(x)=A x \log x+B x, \quad x \in] 0,1],
$$

where $A, B$ are constants, that is,

$$
g(x)=A x \log x+B x+d, \quad x \in] 0,1] .
$$

But, obviously (3.5) holds for $x=0$ in view of (3.3) and the fact that $0 \log 0=0$.

Now, $g$ given by (3.5) is a solution of (1.1), provided $B=(m n-m-n) d$. Thus we have proved the following theorem.

Theorem 1. Let $f:[0,1] \rightarrow \boldsymbol{R}$, be measurable and satisfy (1.1) for a fixed pair $m, n$ $(\geqq 3)$. Then $f$ is a solution of (1.1) if and only if

$$
f(x)=A x \log x+d(m n-m-n) x+d,
$$

where $A, d$ are constants.

Now, let us treat the remaining case when $c \neq 0$ in (3.2) (i.e. the equation (1.3)).

With $y=1,(3.2)$ gives 


$$
c[g(1)+(n-1) d] g(x)=x[d m(n-1)-(1+m c d)(g(1)+(n-1) d)]+d c(g(1)+(n-1) d) .
$$

Hence, when $g(1)+(n-1) d \neq 0$, we obtain

$$
g(x)=\frac{1}{c}\left[\frac{d m(n-1)}{\alpha}-(1+m c d)\right] x+d, \quad x \in[0,1]
$$

where

$$
\alpha=g(1)+(n-1) d .
$$

Now, $g$ given by (3.8) is a solution of (1.3), provided

$$
c \alpha^{2}+\alpha[1-(n-m) c d]-d m(n-1)=0 .
$$

Finally, when $g(1)+(n-1) d=0$, by taking $x_{1}=1=y_{1}, x_{2}=0=\ldots=x_{m}=y_{2}=\ldots=y_{n}$ in (1.3), we get $g(1)=0=g(0)$, so that (3.2) becomes

$$
g(x y)=y g(x)+x g(y)+c g(x) g(y)
$$

which can be reduced to a Cauchy equation

where

$$
h(x y)=h(x) h(y), \text { for } x, y \in] 0,1]
$$

$$
\left.\left.h(x)=1+c \frac{g(x)}{x}, \quad x \in\right] 0,1\right]
$$

Now the solutions of (3.11) are given by

$$
\left.\left.h(x)=0 \quad(x \in] 0,1]), \quad \text { or } \quad h(x)=x^{\beta} \quad(x \in] 0,1\right]\right)
$$

with the convention $1^{\beta}=1$. yields

The solution $h(x)=0$ is not possible, since $h(1)=1$ by (3.12) and $g(1)=0$. Thus (3.12)

$$
g(x)=\frac{x\left(x^{\beta}-1\right)}{c}
$$

for $x \in] 0,1]$. Evidently, since $g(0)=0,(3.13)$ holds for $x=0$ also, provided $\beta \neq-1$. But the solution corresponding to $\beta=-1$ is contained in the solution (3.8) for $d=1 / c$, $g(1)=0$. (The solution is surprisingly independent of $m$ and $n$ ). Thus, we have proved the following theorem.

Theorem 2. Let $g:[0,1] \rightarrow \boldsymbol{R}$ be measurable. Then $g$ is a solution of the equation (1.3) holding for fixed $m, n \geqq 3$ if and only if $g$ has either the form (3.8) with the condition (3.10) or the form (3.13).

Remark 2. If $d=g(0)=0$ and $g(1) \neq 0$, then (3.8) yields

$$
g(x)=-\frac{x}{c}
$$

(a solution independent of $m$ and $n(3,11)$ ). For examples, the solutions of (1.3) (arising from (3.8)) are $g(x)=-10 x+2$ or $g(x)=-3 x+2$, when $m=3=n, c=1, d=2$; $g(x)=10 x-2$ or $g(x)=3 x-2$ when $m=3=n, c=-1, d=-2$ etc. 
But there exists no solution of (1.3) (arising from (3.8)) when $m=3=n, c=-1, d=2$ or $m=3=n, c=1 ; d=-2$ etc for the condition (3.10) is not satisfied in either case; $\alpha^{2}-\alpha+12=0$ and $\alpha^{2}+\alpha+12=0$ have no real solutions for $\alpha(=g(1)+2 d)$.

Remark 3. If the equation (1.1) holds for all $m$ and $n$, it follows from Theorem 1, (3.6), that $d=0$ and the measurable solution of (1.1) takes the form $f(x)=A x \log x$.

If the equation (1.3) holds for all $m$ and $n$, then the measurable solutions of (1.3) are given by $g(x)=-x / c$ or by (3.12) $g(x)=\left(x^{\delta+1}-x\right) / c$. For, the equation (3.10) for $m=n$ gives $c \alpha^{2}+\alpha-d m(m-1)=0$, which for any two distinct values of $m$ yields $d=0$ so that the solution of (1.3) corresponding to (3.8) becomes $g(x)=-x / c$ and the other solution is given by (3.12).

Whereas the solution (3.13) of (1.3) is known $(3,11)$, the solution (3.8) of (1.3) involving $m$ and $n$ is new. Further the solution of (1.3) is obtained in Theorem 2 for fixed pair $m, n$ and under the weaker regularity condition on $g$ i.e. under the Lebesgue measurability of $g$.

\section{REFERENCES}

(1) J. ACZÉl und Z. DARÓCZY, Charakterisierung der Entropien positiver ordnung und der Shannonschen Entropie, Acta Math. Acad. Sci. Hungar. 14 (1963), 95-121.

(2) M. BEHARA, Additive and non-additive measures of entropy with applications (McMaster University report, 1978).

(3) M. BEHARA and P. NATH, Additive and non-additive entropies of finite measurable partitions, Probability and Information Theory, Vol. 2, Springer-Verlag, 1973, V296, 102-138. 7-8.

(4) T. W. Chaundy and J. B. MCLEOD, On a functional equation, Edin. Math. Notes 43(1960),

(5) DarózY, Über die charakterisierung der Shannonschen Entropy, Proc. Colloq. on Information Theory, I, Jóınıss I 3o ly ai Math. Soc., Budapest (1968), 135-139.

(6) Z. DARÓCZY, On the measurable solutions of a functional equation, Acta Math. Acad. Sci. Hungar. 22 (1971), 11-14.

(7) J. HAVRda and F. Charvat, Quantification method of classification process, Concept of structural a-entropy, Kybernetika (Prague), 3 (1967), 30-35.

(8) Pl. Kannappan, On Shannon's entropy, directed divergence and inaccuracy, Z. Wahr. Verw. Geb. 22 (1972), 95-100.

(9) Pl. Kannappan, On directed divergence and inaccuracy, Z. Wahr. Verw. Geb. 25 (1972), 49-55.

(10) Pl. KANNAPPan, On a functional equation connected with generalized directed divergence, Aeq. Math. (1974), 81-93.

(11) Pl. KannaPpan, On generalization of some measures in information theory, Glasnik Mat. 29 (1974), 81-93.

(12) Pl. Kannappan, On a generalization of sum form functional equation -1 (submitted).

(13) C. T. NG, Representation of measures of information with the branching property, Information and Control 25 (1974), 45-56.

UNIVERSITY OF WATERLOO

WATERLOO, ONTARIO 